\title{
Article \\ Effects of Coupling Water and Fertilizer on Agronomic Traits, Sugar Content and Yield of Sugarcane in Guangxi, China
}

\author{
Weixiong Wu ${ }^{1,2,3}$, Wuxiang Fu ${ }^{4,5}$, Juha M. Alatalo ${ }^{6}$, Zhanxia Ma ${ }^{4,7, *}$ and Yang Bai ${ }^{4,8, *}$ \\ 1 School of Water Conservancy and Hydropower, Hebei University of Engineering, Handan 056038, China; \\ solowoo@126.com \\ 2 Guangxi Key Laboratory of Water Engineering Materials and Structures, Nanning 530023, China \\ 3 Guangxi Water Conservancy Research Institute, Nanning 530023, China \\ 4 Center for Integrative Conservation, Xishuangbanna Tropical Botanical Garden, Chinese Academy of Sciences, \\ Xishuangbanna 666303, China; fuwuxiang@163.com \\ 5 College of Agronomy and Biotechnology, Yunnan Agricultural University, Kunming 650201, China \\ 6 Environmental Science Center, Qatar University, Doha 2713, Qatar; jalatalo@qu.edu.qa \\ 7 College of Tea (Pu'er), West Yunnan University of Applied Sciences, Pu'er 665000, China \\ 8 Center of Conservation Biology, Core Botanical Gardens, Chinese Academy of Sciences, Mengla 666303, China \\ * Correspondence: mazhanxia@xtbg.ac.cn (Z.M.); youngbcs@gmail.com (Y.B.)
}

check for updates

Citation: Wu, W.; Fu, W.; Alatalo, J.M.; Ma, Z.; Bai, Y. Effects of Coupling Water and Fertilizer on Agronomic Traits, Sugar Content and Yield of Sugarcane in Guangxi, China. Agronomy 2022, 12, 321. https:// doi.org/10.3390/agronomy12020321

Academic Editors: Jongkwon Im, Xuwang Zhang, Laura Bulgariu, Christos S. Akratos and

Francisco Osorio

Received: 15 December 2021

Accepted: 24 January 2022

Published: 26 January 2022

Publisher's Note: MDPI stays neutral with regard to jurisdictional claims in published maps and institutional affiliations.

Copyright: (C) 2022 by the authors. Licensee MDPI, Basel, Switzerland. This article is an open access article distributed under the terms and conditions of the Creative Commons Attribution (CC BY) license (https:// creativecommons.org/licenses/by/ $4.0 /)$.

\begin{abstract}
This work is a contribution to applied water and fertilizer coupling efforts aiming at increasing crop productivity on sugarcane plantations through the analysis of average cane yields obtained following main agronomic traits in growth and sugar content of plantations, in order to improve irrigation scheduling practices. A field experiment was carried out, testing drip irrigation (D), with four levels $(0 \%, 50 \%, 100 \%$ and $150 \%)$ of water $(\mathrm{W})$ and four levels $(0 \%, 50 \%, 100 \%$ and $150 \%$ ) of fertilizer (F) with three replicates. Rain-fed crop (neither irrigation nor fertilizer DW0F0) and manpower irrigation were included as the control (W1F1). The effects of water and fertilizer on agronomic traits (plant high, stem diameter, effective stem number and single stem weight), sugarcane yield and sugar content were studied in Guangxi (southwest China) from 2018-2020. Compared with W1F1, the agronomic traits were higher under DW1F1 treatment; for sugar content, the benefit of the combined treatment was W0.5F0; for sugarcane yield, the best treatment was DW1F1, followed by DW1.5F1. To obtain maximum sugar yield, it is recommended to apply a N fertilizer dose of $292 \mathrm{~kg} \mathrm{ha}^{-1}, \mathrm{~K}_{2} \mathrm{O}$ fertilizer $146.55 \mathrm{~kg} \mathrm{ha}^{-1}$ and $\mathrm{P}_{2} \mathrm{O}_{5}$ fertilizer $439.5 \mathrm{~kg} \mathrm{ha}^{-1}$ with water $1778.4 \mathrm{~m}^{3} \mathrm{ha}^{-1}$. The results could not only boost efficiency of water and fertilizer, but also establish the reasonable irrigation and fertilizer measure, and regulate yield of sugarcane. It could offer some ideas and techniques for developing precision farming.
\end{abstract}

Keywords: water-fertilizer coupling; drip irrigation; sugar yield; precision farming

\section{Introduction}

Sugarcane (Saccharum officinarum L.) is an important crop, of significant social and economic value in many countries, given that nearly $75 \%$ of global production is concentrated in Brazil, India, China, Thailand, and Pakistan [1,2]. Sugarcane plays a pivotal role in both the agricultural and industrial economy of China as it is a renewable natural agricultural resource and provides sugar as well as biofuel, fiber and co-products with ecological sustainability [3,4]. Guangxi is an important sugarcane production and processing base in China; its planting area accounts for more than $85 \%$ of the domestic annual sugarcane planting area, and its sugar production accounts for more than $90 \%$ of the total sugar output [5].

Water is essential for the growth of sugarcane, and water deficiency can seriously retard sugarcane's development [6,7]. Sugarcane responds differently to a water deficit during different growth phases. In the early stages of sugarcane growth, a water deficit 
will lead to compensatory growth of the plant when the normal water level is restored. Therefore, in the early stage of sugarcane growth, water can be appropriately saved [8-10]. Studies have clearly shown that reducing irrigation during the maturation phase saved water and increased the sugar content $[11,12]$. Contrary to the initial and final stages of sugarcane development, many studies have shown that precipitation has the greatest impact on sugarcane elongation. Lack of water often leads to reduced sugarcane production and even failed harvests [13,14].

Sugarcane is a high-yielding crop that requires significant amounts of plant nutrients, because mineral elements comprise $3-5 \%$ of its dry matter. Sugarcane accumulates, per $100 \mathrm{mg}$ of stalks produced, $100-154 \mathrm{~kg} \mathrm{~N}$; $15-25 \mathrm{~kg} \mathrm{P}_{2} \mathrm{O}_{5}$; 77-232 $\mathrm{kg} \mathrm{K} \mathrm{K}_{2} \mathrm{O}$; and $14-49 \mathrm{~kg} \mathrm{~S}$ [15]. This amount of nutrients might be replaced every year to allow sustainable production with no degradation of soil fertility over the years [16]. Due to insufficient fertilizers, the growth and yield of sugarcane has declined as it is required in many critical processes indicating plant growth such as leaves expansion and the production of tiller or sucker, especially in the formation of plant protein; however, excessive fertilizer (especially nitrogen) can lead to prolonged plant growth and reduced sugar yield and quality [17]. In addition, because fertilizer requires substantial input costs and has an impact on the environment, there are pressing needs to optimize the supply of fertilizer with the most critical crop requirement stages [4]. As a consequence, a considerable ratio of the production cost of sugarcane is associated with the use of soil amendments and fertilizers [18].

In combination, water and nutrients are the important factors in agricultural growth and production, and different levels of water and fertilizers have significant combined effects on yield, crop growth and water productivity [19]. For different varieties, regional climate, soil and other conditions, the water and fertilizer supply parameters will also vary. However, at present, there are not many reports on the water and fertilization integration technology in sugarcane. Excessive irrigation water and fertilizers not only waste resources, but also seriously affect the environment [20-24]. Therefore, it is necessary to find a reasonable coupling water and fertilizer models that allows the highest productivity for sugarcane. A large amount of sugarcane is planted on dry slopes in Guangxi, coupled with the typical karst landform features, making it difficult to store water in planting areas. Studies have shown that drought and water shortage has become the main constraint factor for the high and stable production of sugarcane in Guangxi $[25,26]$. Therefore, to achieve maximum productivity, it requires an abundant supply of water from effective irrigation, which also can contribute to sustainable agricultural development and environmental sustainability. Substantial amounts of fertilizer are necessary for high sugarcane production, due to the crop's high biomass production [3]. However, studies on the effects of fertilization on sugarcane biomass and sugar accumulation have revealed that high fertilization is a strong source of greenhouse gases emissions [27], meaning it has both economic and environmental consequences.

We monitored agronomic traits, sugar content and yield traits of sugarcane from 2018-2020 under different water-fertilization management systems in a typical sugarcane cropping system. The aim of this study is to optimize water and fertilizer application to maximize sugar content and optimize the yield traits of sugarcane to improve the benefits, which can provide a scientific basis for optimization and management of irrigation and fertilization in China and other regions with similar environments.

\section{Materials and Methods}

\subsection{Study Area}

The study area was in Jiangzhou county, Guangxi province, a typical sugarcaneproducing area in China (Figure 1). Jiangzhou county is located in the subtropical humid monsoon climate zone, with mean annual precipitation of $1500 \mathrm{~mm}$ and mean annual temperature of $22^{\circ} \mathrm{C}$. Sugarcane in Guangxi plays a crucial role in China's sugar production and regional economic development. The area of sugarcane crop increased from 508.7 ha in 2000 to 890.2 ha in 2019 , representing $8.1 \%$ and $14.9 \%$, respectively, of the total sown 
area in Guangxi province [28]. Abundant light and heat resources are of great benefit to the yield of sugarcane. Following adjustment of the planting system, sugar production has been strongly increased in Guangxi.

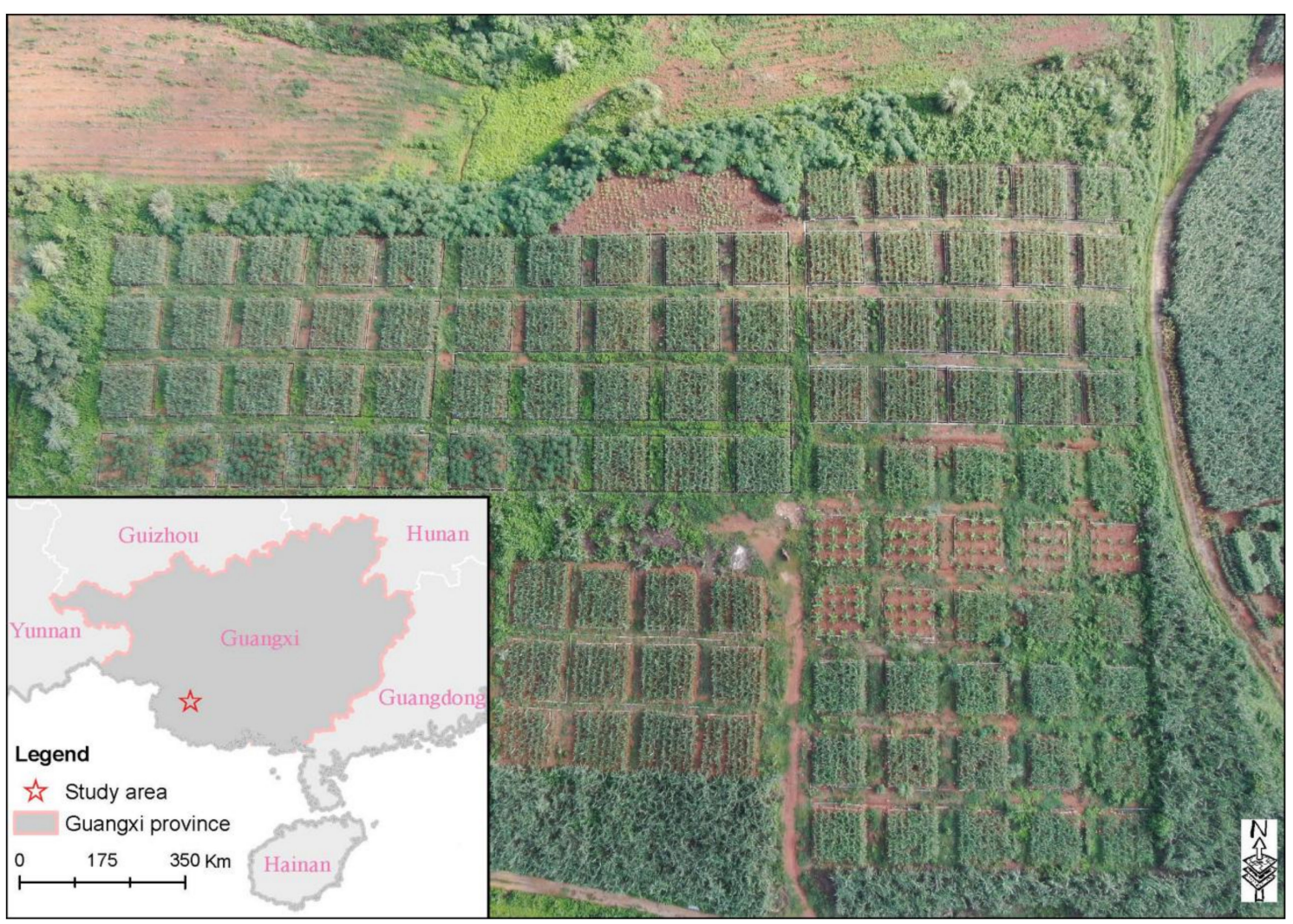

Figure 1. Location of the study site in Guangxi province, southern China, and the experimental sugarcane plot.

\subsection{Experiment Design}

The experiment was carried out at Longze in Jiangzhou county. Rainfall is mainly concentrated from April to October and few showers in December to late spring (Figure 2). The soil was generally low in major nutrients, with $0.5 \mathrm{~g} \cdot \mathrm{kg}^{-1}$ of total N, $57 \mathrm{mg} \mathrm{kg} \mathrm{g}^{-1}$ of available phosphorus (P), $125 \mathrm{mg} \mathrm{kg}{ }^{-1}$ of available potassium (K), and $8.3 \mathrm{~g} \cdot \mathrm{kg}^{-1}$ of organic matter. High ridges are raised before planting, and sugarcane is planted on the ridges. During the growth process of sugarcane, the weeding and spraying of pesticides were carried out according to the actual situation to prevent the occurrence of diseases and insect pests. Weeds may smother new shoots before they grow. Continued weeding is necessary until the cane grows large enough to create shade and block harmful weeds. Checking sugarcane regularly for pests or rot, and taking precautions to keep pests and disease at bay as much as possible are of the utmost importance. Each plot was $8 \mathrm{~m} \times 8 \mathrm{~m}$ and was equipped with one of two irrigation methods-drip (D) or manpower irrigation-and different fertilizer levels. The drip irrigation zone was surrounded by cement and bricks, to form a relatively independent experimental plot. The manpower irrigation zone had a $2 \mathrm{~m}$ wide isolation zone. 


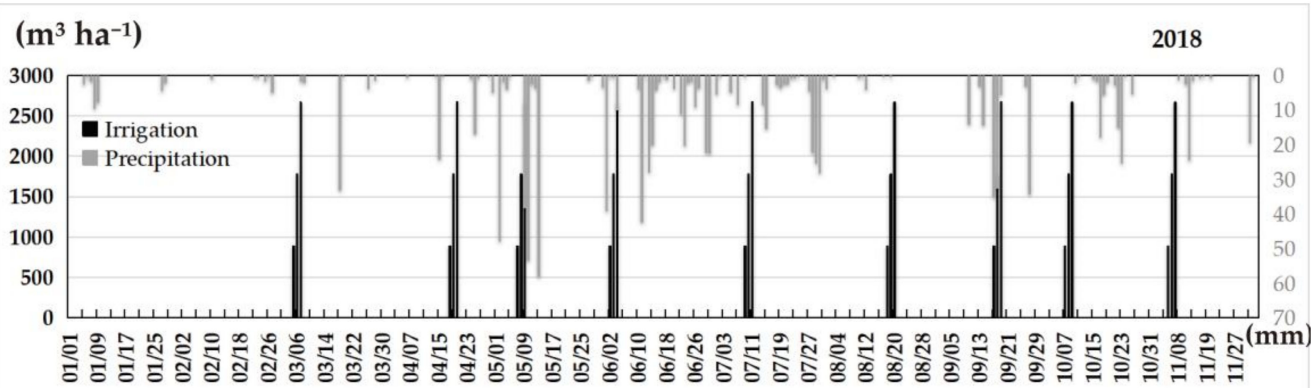

Date(Month-day)

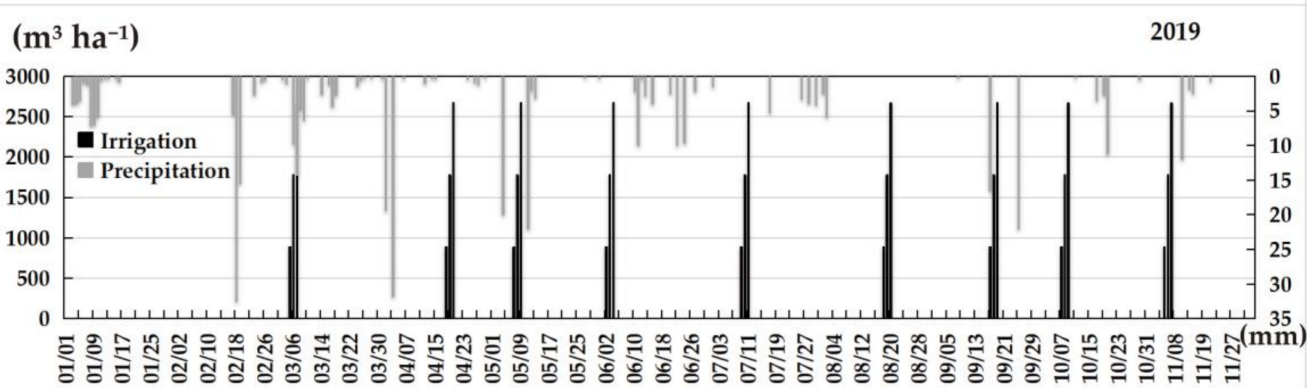

Date(Month-day)

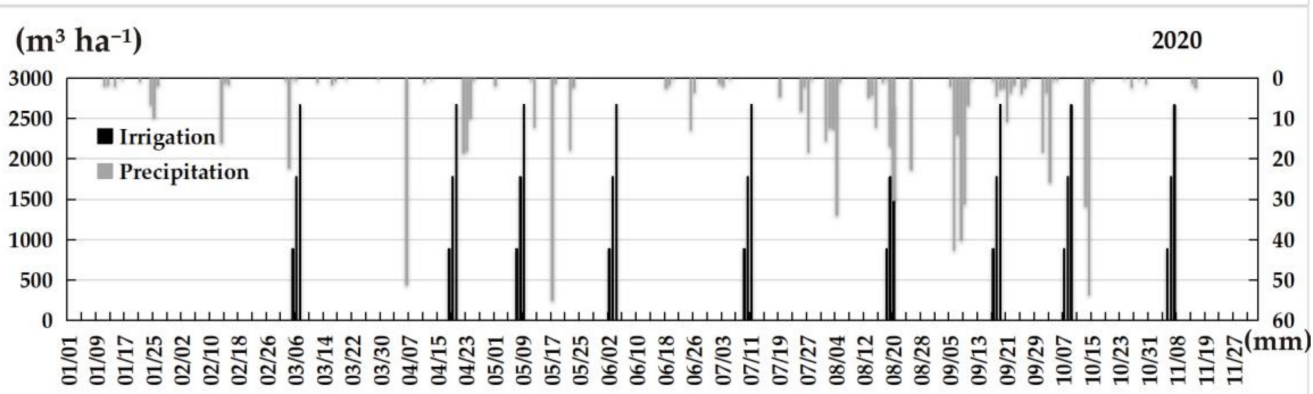

Date(Month-day)

Figure 2. Precipitation and irrigation from January to November in 2018-2020.

The experiments were conducted in a randomized block factorial design with three replicates. The treatments repeated at the same location each year. The experimental treatments comprised four levels of irrigation and four levels of fertilization. In total, 13 treatments were conducted in the experiment, including 12 water and fertilizer coupling treatments, a manpower treatment (W1F1) and the control treatments (CK).

The irrigation application was started at 30 days after planting. Irrigation level was designed according to the lower limit of soil moisture content. The soil moisture content in the seedling and tillering stage was $65-85 \%$ of the field water holding capacity, the elongation was $70-90 \%$ and the maturity period was $60-80 \%$. The irrigation volume was set to four gradients of this level: W0 (0\%), W0.5 (50\%), W1 (100\%) and W1.5 (150\%).

Fertilization is distributed according to the proportion of $10 \%$ in the seedling stage, $20 \%$ in the tillering stage, and $70 \%$ in the elongation stage. The ratio of $\mathrm{N}, \mathrm{K}_{2} \mathrm{O}$ and $\mathrm{P}_{2} \mathrm{O}_{5}$ fertilizer was 1.53:1:3.07. The four levels of $\mathrm{N}, \mathrm{K}_{2} \mathrm{O}$ and $\mathrm{P}_{2} \mathrm{O}_{5}$ fertilizer (0-0-0, 146.55-73.2219.75, 292.95-146.48-439.43 and 439.5-219.75-659.1 kg.ha ${ }^{-1}$ were designated F0, F0.5, F1 and F1.5, respectively) were applied to the plots. The F0, F0.5, F1 and F1.5 treatments represented $0 \%, 50 \%, 100 \%$ and $150 \%$ of the locally applied fertilizer rate (292.95-146.48$439.43 \mathrm{~kg} \cdot \mathrm{ha}^{-1} \mathrm{~N}, \mathrm{~K}_{2} \mathrm{O}$ and $\mathrm{P}_{2} \mathrm{O}_{5}$ ), respectively. The fertilization time of the seedling period was 5 March, the tillering period was 18 April and the elongation period was 2 June each year. The amount of fertilization and irrigation during 2018-2020 is shown in Table 1. 
Table 1. Field experimental design: Drip (D) irrigation, and $0 \%$ (W0), 50\% (W0.5), $100 \%$ (W1), 150\% (W1.5) of the optimal irrigation level, and $0 \%$ (F0), 50\% (F0.5), 100\% (F1) or 150\% (F1.5) of the optimal fertilization level.

\begin{tabular}{|c|c|c|c|c|}
\hline \multirow{2}{*}{ Treatment } & \multirow{2}{*}{ Water Rate $\left(\mathrm{m}^{3} \mathrm{ha}^{-1}\right)$} & \multicolumn{3}{|c|}{ Fertilizer Rate (kg ha $\left.{ }^{-1}\right)$} \\
\hline & & $\mathbf{N}$ & $\mathrm{K}_{2} \mathrm{O}$ & $\mathbf{P}_{2} \mathrm{O}_{5}$ \\
\hline W1F1 (CK) & 1778.4 & 292.95 & 146.48 & 439.43 \\
\hline DWOF0 & 0.00 & 0.00 & 0.00 & 0.00 \\
\hline DW0F0.5 & 0.00 & 146.55 & 73.2 & 219.75 \\
\hline DW0F1 & 0.00 & 292.95 & 146.55 & 439.5 \\
\hline DW0.5F0 & 889.2 & 0.00 & 0.00 & 0.00 \\
\hline DW0.5F0.5 & 889.2 & 146.55 & 73.2 & 219.75 \\
\hline DW0.5F1 & 889.2 & 292.95 & 146.55 & 439.5 \\
\hline DW1F0 & 1778.4 & 0.00 & 0.00 & 0.00 \\
\hline DW1F0.5 & 1778.4 & 146.55 & 73.2 & 219.75 \\
\hline DW1F1 & 1778.4 & 292.95 & 146.55 & 439.5 \\
\hline DW1.5F0.5 & 2667.6 & 146.55 & 73.2 & 219.75 \\
\hline DW1.5F1 & 2667.6 & 292.95 & 146.55 & 439.5 \\
\hline DW1.5F1.5 & 2667.6 & 439.5 & 219.75 & 659.1 \\
\hline
\end{tabular}

\subsection{Trait Measurements}

We referred to the method of "Chinese Sugarcane Varieties" to identify various agronomic indicators of sugarcane. Beginning in June 2018, the plant height, effective stem number, single stem weight and stem diameter sugarcane yield were investigated at late maturity. The sugar content monitoring started from the formation of the first node of the plant, and from each plot 5 representative plants (non-numbered internal plants) were selected to measure the sugar content in the upper, middle and lower positions, starting from the appearance of two nodes, and measuring two, normal measurement after three sessions, the target reaction average sugar content. The field hammers of the sugarcane plant were measured with a handheld hammer every 15 days, and the upper and lower field hammers of the cane stems reached $0.9-1.0$ at the mature stage.

Sugar content $(\%)=$ field hammer degree $\times 1.025-7.703$.

\subsection{Statistical Analysis}

Statistical analyses were performed using the software $\mathrm{R}$ (Version 4.02). All values were expressed as means \pm SD. One-way ANOVA and all pair-wise comparisons of the treatment means were performed using Duncan test, with significance set at $p<0.05$.

\section{Results}

\subsection{Combined Effect of Water and Fertilization on Sugarcane Agronomic Traits}

In 2018, water had a significant effect on plant height $(\mathrm{F}=3.557, p=0.018)$, stem diameter $(\mathrm{F}=2.845, p=0.043)$, effective stem number $(\mathrm{F}=6.483, p<0.001)$ and single stem weight $(\mathrm{F}=4.992, p=0.004)$. Fertilizer and fertilizer-water interaction had no significant effect on all agronomic characteristics of sugarcane $(p>0.05)$. In 2019 , water had a significant effect on effective stem number $(\mathrm{F}=6.089, p=0.001)$ and single stem weight $(\mathrm{F}=5.398, p<0.001)$, and fertilizer had a significant effect on effective stem number $(\mathrm{F}=3.764, p=0.021)$. In 2020, water had a significant effect on effective plant height $(\mathrm{F}=8.197, p<0.001)$ and single stem weight $(\mathrm{F}=5.33, p=0.002)$, fertilizer had a significant effect on plant height $(\mathrm{F}=10.93, p<0.001)$, and fertilizer-water interaction had a significant effect on plant height $(\mathrm{F}=4.22, p=0.005)$ and single stem weight $(\mathrm{F}=3.22, p=0.020)$ (Table 2). 
Table 2. Effect of different water and fertilizer treatments on sugarcane major agronomic traits in 2018-2020.

\begin{tabular}{|c|c|c|c|c|c|}
\hline Years & Treatment & Plant Height & Stem Diameter & Effective Stem Number & Single Stem Weight \\
\hline \multirow{13}{*}{2020} & W1F1 & $234.63 \pm 16.86 \mathrm{de}$ & $27.02 \pm 1.65 \mathrm{a}$ & $344.67 \pm 3.51 \mathrm{abc}$ & $1.09 \pm 0.11 \mathrm{def}$ \\
\hline & DW0F0 & $248.37 \pm 7.83$ cde & $25.44 \pm 1 \mathrm{a}$ & $346.67 \pm 44.06 \mathrm{abc}$ & $1.12 \pm 0.04 \mathrm{cdef}$ \\
\hline & DW0F0.5 & $227.4 \pm 8.07 \mathrm{e}$ & $26.82 \pm 0.91 \mathrm{a}$ & $341.67 \pm 64.39 \mathrm{abc}$ & $1 \pm 0.1$ ef \\
\hline & DW0F1 & $258.6 \pm 12.6 \mathrm{bcd}$ & $26.88 \pm 1.75 \mathrm{a}$ & $326.67 \pm 11.55 \mathrm{bc}$ & $1.15 \pm 0.08$ bcde \\
\hline & DW0.5F0 & $228.43 \pm 14.31 \mathrm{e}$ & $26.31 \pm 0.23 \mathrm{a}$ & $316 \pm 38.3 \mathrm{bc}$ & $1.26 \pm 0.23 \mathrm{abcd}$ \\
\hline & DW0.5F0.5 & $284.63 \pm 14.47 \mathrm{ab}$ & $26.19 \pm 0.75 \mathrm{a}$ & $353.67 \pm 56.2 \mathrm{abc}$ & $1.16 \pm 0.05$ bcde \\
\hline & DW0.5F1 & $281.7 \pm 25.77 \mathrm{ab}$ & $27.4 \pm 1.82 \mathrm{a}$ & $329.67 \pm 30.92 a b c$ & $1.22 \pm 0.12 \mathrm{abcd}$ \\
\hline & DW1F0 & $240.23 \pm 13.54$ cde & $25.69 \pm 0.44 \mathrm{a}$ & $309.67 \pm 17.04 c$ & $0.91 \pm 0.21 \mathrm{f}$ \\
\hline & DW1F0.5 & $279.57 \pm 0.65 \mathrm{ab}$ & $25.88 \pm 0.31 \mathrm{a}$ & $386 \pm 49.39 \mathrm{a}$ & $1.26 \pm 0.13 \mathrm{abcd}$ \\
\hline & DW1F1 & $299.93 \pm 4.01 \mathrm{a}$ & $27.26 \pm 0.72 \mathrm{a}$ & $363.33 \pm 17.56 \mathrm{abc}$ & $1.32 \pm 0.12 \mathrm{abc}$ \\
\hline & DW1.5F0.5 & $263.37 \pm 18.15 \mathrm{bc}$ & $27.11 \pm 0.39 \mathrm{a}$ & $366.67 \pm 10.97 \mathrm{ab}$ & $1.21 \pm 0.03$ abcde \\
\hline & DW1.5F1 & $279.87 \pm 22.05 a b$ & $25.74 \pm 1.16 \mathrm{a}$ & $363.83 \pm 24.91 \mathrm{ab}$ & $1.38 \pm 0.11 \mathrm{a}$ \\
\hline & DW1.5F1.5 & $279.7 \pm 16.79 \mathrm{ab}$ & $25.8 \pm 2.12 \mathrm{a}$ & $370.33 \pm 10.02 \mathrm{ab}$ & $1.33 \pm 0.17 \mathrm{ab}$ \\
\hline \multirow{13}{*}{2019} & W1F1 & $182.67 \pm 3.37 \mathrm{~b}$ & $25.95 \pm 0.25 \mathrm{ab}$ & $311.67 \pm 52.25$ cde & $0.81 \pm 0.09 \mathrm{abcd}$ \\
\hline & DW0F0 & $190.63 \pm 15.99 \mathrm{~b}$ & $27.22 \pm 0.63 \mathrm{a}$ & $286.33 \pm 40.82 \mathrm{e}$ & $0.67 \pm 0.06 \mathrm{de}$ \\
\hline & DW0F0.5 & $192.53 \pm 12.45 b$ & $26.12 \pm 0.85 \mathrm{ab}$ & $308.67 \pm 48.52$ cde & $0.72 \pm 0.11$ bcde \\
\hline & DW0F1 & $188.63 \pm 10.68 b$ & $25.46 \pm 0.74 \mathrm{~b}$ & $297.33 \pm 30.02$ cde & $0.74 \pm 0.07$ bcde \\
\hline & DW0.5F0 & $196.03 \pm 4.2 \mathrm{~b}$ & $26.55 \pm 1.03 \mathrm{ab}$ & $322 \pm 0$ bcde & $0.7 \pm 0.05$ cde \\
\hline & DW0.5F0.5 & $287.1 \pm 116.4 \mathrm{a}$ & $26.75 \pm 0.87 \mathrm{ab}$ & $374.67 \pm 26.69 a b$ & $0.82 \pm 0.06 \mathrm{abcd}$ \\
\hline & DW0.5F1 & $206.6 \pm 16.71 \mathrm{~b}$ & $25.34 \pm 0.7 \mathrm{~b}$ & $353 \pm 28.79 a b c$ & $0.82 \pm 0.09 \mathrm{abcd}$ \\
\hline & DW1F0 & $190.38 \pm 12.69 b$ & $25.58 \pm 1.52 b$ & $292.33 \pm 34.36 \mathrm{de}$ & $0.64 \pm 0.06 \mathrm{e}$ \\
\hline & DW1F0.5 & $211.2 \pm 13.13 \mathrm{~b}$ & $25.39 \pm 0.84 \mathrm{~b}$ & $350 \pm 9.54 \mathrm{abcd}$ & $0.88 \pm 0.09 \mathrm{ab}$ \\
\hline & DW1F1 & $201.7 \pm 9.42 b$ & $25.94 \pm 0.47 \mathrm{ab}$ & $378.33 \pm 62.82 \mathrm{ab}$ & $0.83 \pm 0.03 \mathrm{abcd}$ \\
\hline & DW1.5F0.5 & $199 \pm 7.57 \mathrm{~b}$ & $26.15 \pm 1.46 \mathrm{ab}$ & $343.33 \pm 19.66$ abcde & $0.86 \pm 0.13 \mathrm{abc}$ \\
\hline & DW1.5F1 & $217.52 \pm 21.39 b$ & $25.91 \pm 1.14 \mathrm{ab}$ & $372.67 \pm 32.66 \mathrm{ab}$ & $0.94 \pm 0.14 \mathrm{a}$ \\
\hline & DW1.5F1.5 & $206.03 \pm 3.78 b$ & $25.49 \pm 1.02 \mathrm{~b}$ & $400 \pm 39.85 \mathrm{a}$ & $0.87 \pm 0.12 \mathrm{ab}$ \\
\hline \multirow{13}{*}{2018} & W1F1 & $300.07 \pm 19.7 \mathrm{ab}$ & $27.61 \pm 2.2 \mathrm{ab}$ & $364.67 \pm 51.25 \mathrm{abc}$ & $1.44 \pm 0.04 \mathrm{bc}$ \\
\hline & DW0F0 & $312.37 \pm 6.46 \mathrm{a}$ & $28.1 \pm 0.65 \mathrm{ab}$ & $404 \pm 11.53 \mathrm{a}$ & $1.46 \pm 0.03 b c$ \\
\hline & DW0F0.5 & $308 \pm 2.17 \mathrm{a}$ & $28.82 \pm 1.62 \mathrm{a}$ & $379.33 \pm 13.05 \mathrm{ab}$ & $1.47 \pm 0.06 \mathrm{bc}$ \\
\hline & DW0F1 & $287.7 \pm 8.68 \mathrm{ab}$ & $27.99 \pm 0.59 \mathrm{ab}$ & $390.67 \pm 16.92 \mathrm{ab}$ & $1.42 \pm 0.04 \mathrm{bcd}$ \\
\hline & DW0.5F0 & $278 \pm 33.59 \mathrm{~b}$ & $27.11 \pm 2.95 \mathrm{ab}$ & $318.33 \pm 23.09 c$ & $1.23 \pm 0.12 \mathrm{~d}$ \\
\hline & DW0.5F0.5 & $287.63 \pm 17.93 a b$ & $26.26 \pm 1.56 \mathrm{~b}$ & $348.33 \pm 39.46 \mathrm{bc}$ & $1.31 \pm 0.01 \mathrm{~cd}$ \\
\hline & DW0.5F1 & $279 \pm 18.56 \mathrm{~b}$ & $26.24 \pm 1.27 \mathrm{~b}$ & $347.33 \pm 13.65 \mathrm{bc}$ & $1.4 \pm 0.23 \mathrm{bcd}$ \\
\hline & DW1F0 & $302.97 \pm 16.1 \mathrm{ab}$ & $28.13 \pm 1.41 \mathrm{ab}$ & $345 \pm 30 \mathrm{bc}$ & $1.5 \pm 0.18 \mathrm{abc}$ \\
\hline & DW1F0.5 & $312.4 \pm 11.18 \mathrm{a}$ & $28.38 \pm 0.93 \mathrm{ab}$ & $384.33 \pm 61.45 a b$ & $1.54 \pm 0.13 \mathrm{ab}$ \\
\hline & DW1F1 & $298.3 \pm 17.04 \mathrm{ab}$ & $28.44 \pm 0.53 \mathrm{ab}$ & $415.33 \pm 28.45 \mathrm{a}$ & $1.66 \pm 0.1 \mathrm{a}$ \\
\hline & DW1.5F0.5 & $296.6 \pm 5.39 \mathrm{ab}$ & $29.22 \pm 0.38 \mathrm{a}$ & $397.33 \pm 35.3 \mathrm{ab}$ & $1.4 \pm 0.13 \mathrm{bcd}$ \\
\hline & DW1.5F1 & $303.67 \pm 14.38 \mathrm{a}$ & $28.28 \pm 1.09 \mathrm{ab}$ & $410.17 \pm 22.25 \mathrm{a}$ & $1.49 \pm 0.15 \mathrm{bc}$ \\
\hline & DW1.5F1.5 & $311.43 \pm 11.83 \mathrm{a}$ & $27.71 \pm 0.84 \mathrm{ab}$ & $389.33 \pm 40.7 \mathrm{ab}$ & $1.53 \pm 0.04 \mathrm{ab}$ \\
\hline
\end{tabular}

Different letters- $\mathrm{a}, \mathrm{b}, \mathrm{c}, \mathrm{d}$, e and $\mathrm{f}$-in the table indicate significant differences between treatments.

Integrating the data of 2018-2020 on sugarcane agronomic traits, as shown in Table 2, among different treatments, the treatments DW1F1, DW1.5F1 and DW1.5F1.5 were better for effective stem number and single stem weight, which are the main agronomic yield traits for sugarcane (Figure 3). Water, fertilizer and water-fertilizer interaction had significant effects on mean plant height, mean effective stem number and mean single stem weight in 3 years, and had no significant on mean stem diameter (Table 3, Figure 4). 

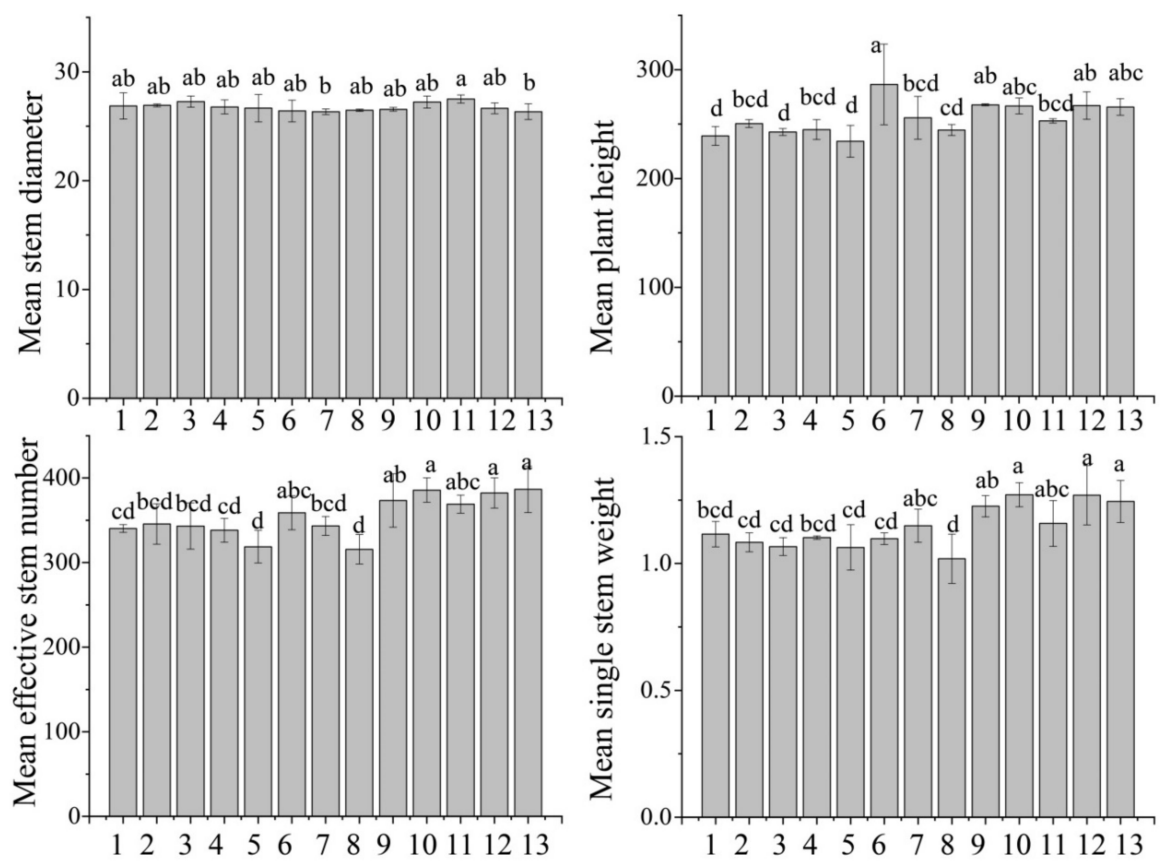

Figure 3. Effect of different water and fertilizer treatments on mean sugarcane major agronomic traits in 2018-2020. (Different letters-a, b, c and d-in the figure indicate significant differences between treatments. 1-W1F1; 2-DW0F0; 3-DW0F0.5; 4-DW0F1; 5-DW0.5F0; 6-DW0.5F0.5; 7-DW0.5F1; 8-DW1F0; 9-DW1F0.5; 10-DW1F1; 11-DW1.5F0.5; 12-DW1.5F0.5; 13-DW1.5F1.5).
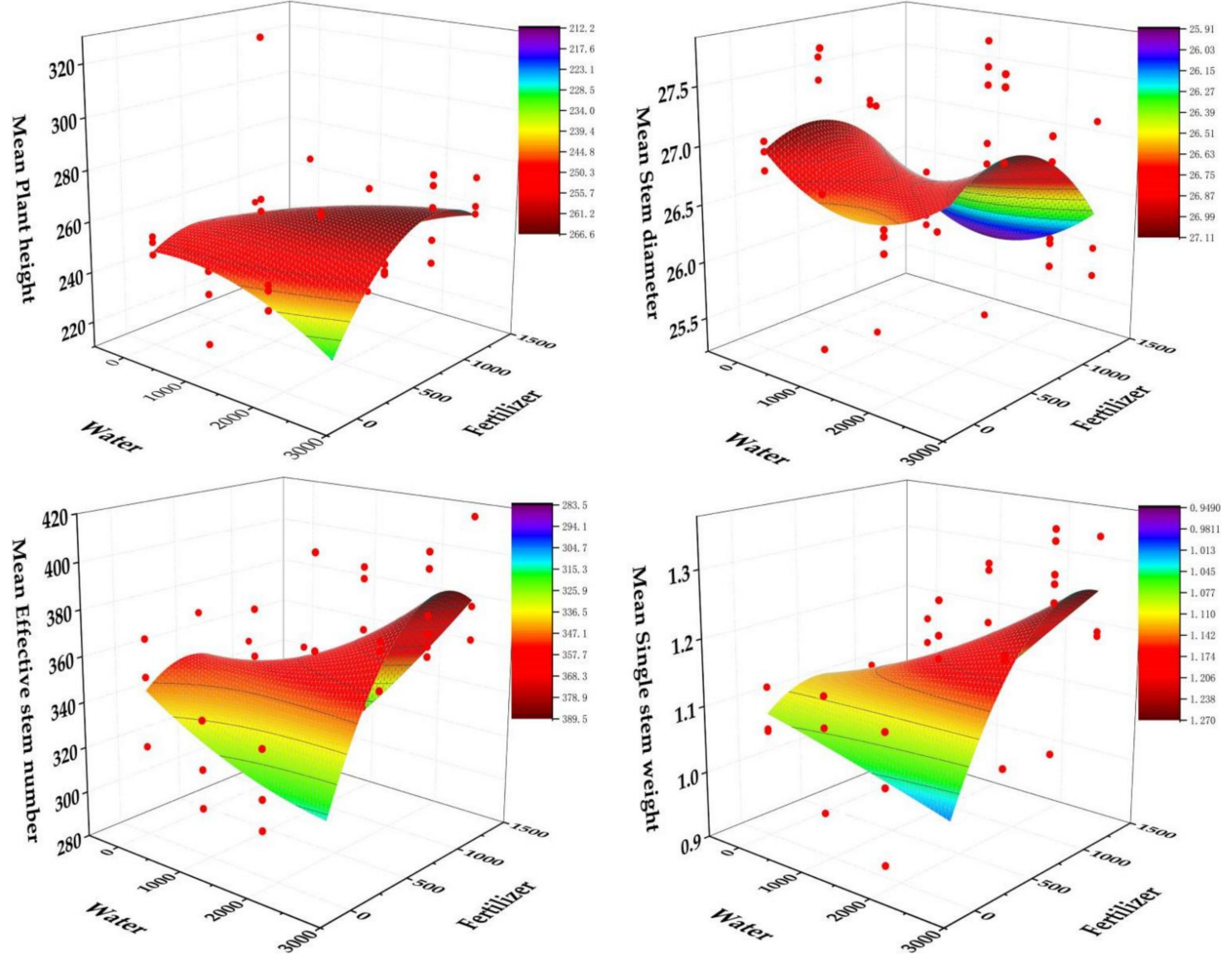

Figure 4. Regression equation of mean plant height, mean stem diameter, mean effective stem number and mean single stem weight with irrigation and fertilization. 
Table 3. Results of two-way ANOVA test (F) on major agronomic traits of sugarcane under different water-fertilizer irrigation treatment methods from 2018-2020 (mean represents the 3-year average of agronomic traits).

\begin{tabular}{|c|c|c|c|c|c|c|c|c|c|c|c|c|c|c|c|c|}
\hline & \multicolumn{4}{|c|}{ Plant Height } & \multicolumn{4}{|c|}{ Stem Diameter } & \multicolumn{4}{|c|}{ Effective Stem Number } & \multicolumn{4}{|c|}{ Single Stem Weight } \\
\hline & 2018 & 2019 & 2020 & Mean & 2018 & 2019 & 2020 & Mean & 2018 & 2019 & 2020 & Mean & 2018 & 2019 & 2020 & Mean \\
\hline Water & 3.56 * & 2.11 & $8.20 * * *$ & $3.44 *$ & 2.85 * & 0.65 & 0.51 & 0.72 & $6.48 * * *$ & $6.10^{* *}$ & 1.53 & $7.50 * * *$ & $4.99 * *$ & $5.40 *$ & $5.33 * * *$ & $6.89 * * *$ \\
\hline Fertilizer & 1.29 & 1.42 & $10.93^{* * *}$ & $3.47 *$ & 0.415 & 1.42 & 1.36 & 0.89 & 2.67 & $3.76^{*}$ & 1.64 & $4.97 * *$ & 1.19 & 3.80 & 2.68 & 5.02 ** \\
\hline Water $\times$ Fertilizer & 0.99 & 2.10 & $4.22 * *$ & 4.03 ** & $\begin{array}{l}0.415 \\
0.32\end{array}$ & $\begin{array}{l}1.42 \\
1.13\end{array}$ & $\begin{array}{l}1.00 \\
1.40\end{array}$ & 1.18 & 1.42 & $\begin{array}{l}.70 \\
0.98\end{array}$ & $\begin{array}{l}1.04 \\
1.14\end{array}$ & $2.94 *$ & $\begin{array}{l}1.19 \\
0.69\end{array}$ & 0.78 & $3.22^{*}$ & 2.25 * \\
\hline
\end{tabular}

Values are significant at 0.05 level; $^{*},{ }^{* *}$ and ${ }^{* * *}$ refer to significant difference at $0.05,0.01$ and 0.001 probability levels, respectively.

\subsection{Combined Effect of Water and Fertilization on Sugar Content and Sugar Yield}

One-way analysis of variance showed that there was no significant difference in sugarcane field hammer in $2018(\mathrm{~F}=0.150, p=0.961)$, among which the field hammer was the highest under the treatment of DW1.5F0.5; however, there was significant difference among the treatments in $2019(\mathrm{~F}=3.812, p=0.013)$ - the CK (W1F1) treatment was the lowest, and DW0F0 was the highest. There was no significant difference in the sugarcane field hammer among the treatments in $2020(\mathrm{~F}=0.926, p=0.462)$, where DW1.5F0.5 was the highest and DW0.5F0.5 was the lowest. Considering the 3 years comprehensively, the field hammer was the highest under the treatment of DW1.5F0.5 (Table 3, Figure 4). The results of the two-way ANOVA analysis showed that water, fertilizer and water-fertilizer interaction had no significant effect on field hammer (Table 4).

Table 4. Effect of different water and fertilizer treatments on field hammer, sugarcane stem yield and sugar content.

\begin{tabular}{|c|c|c|c|c|}
\hline Year & Treatment & Field Hammer & Sugarcane Stem Yield & Sucrose Content \\
\hline \multirow{13}{*}{2018} & DWOF0 & $21.44 \pm 0.31 \mathrm{a}$ & $588.61 \pm 7.8 \mathrm{abc}$ & $15.5 \pm 0.33 \mathrm{a}$ \\
\hline & DW0F0.5 & $22 \pm 0.37 \mathrm{a}$ & $558.96 \pm 36.97 \mathrm{bcd}$ & $16.11 \pm 0.41 \mathrm{a}$ \\
\hline & DW0F1 & $21.53 \pm 0.46 \mathrm{a}$ & $555.2 \pm 36.07 \mathrm{bcd}$ & $15.61 \pm 0.5 \mathrm{a}$ \\
\hline & DW0.5F0 & $21.86 \pm 1.03 \mathrm{a}$ & $392.74 \pm 69.81 \mathrm{e}$ & $16.47 \pm 1.25 \mathrm{a}$ \\
\hline & DW0.5F0.5 & $21.33 \pm 0.48 \mathrm{a}$ & $457.85 \pm 52.8 \mathrm{de}$ & $15.39 \pm 0.52 \mathrm{a}$ \\
\hline & DW0.5F1 & $21.91 \pm 0.19 \mathrm{a}$ & $489.66 \pm 93.73$ cde & $16.01 \pm 0.2 \mathrm{a}$ \\
\hline & DW1F0 & $21.54 \pm 1.35 \mathrm{a}$ & $520.92 \pm 106.17 \mathrm{bcd}$ & $15.61 \pm 1.46 \mathrm{a}$ \\
\hline & DW1F0.5 & $22.01 \pm 0.8 \mathrm{a}$ & $596.54 \pm 145.32 \mathrm{abc}$ & $16.13 \pm 0.87 \mathrm{a}$ \\
\hline & DW1F1 & $21.56 \pm 0.88 \mathrm{a}$ & $690.72 \pm 44.97 \mathrm{a}$ & $15.63 \pm 0.95 \mathrm{a}$ \\
\hline & DW1.5F0.5 & $22.21 \pm 0.63 a$ & $558.2 \pm 82.61 \mathrm{bcd}$ & $16.34 \pm 0.68 \mathrm{a}$ \\
\hline & DW1.5F1 & $21.79 \pm 0.82 \mathrm{a}$ & $610.89 \pm 71.36 \mathrm{ab}$ & $15.88 \pm 0.89 \mathrm{a}$ \\
\hline & DW1.5F1.5 & $21.61 \pm 0.77 \mathrm{a}$ & $596.19 \pm 49.38 \mathrm{abc}$ & $15.69 \pm 0.84 \mathrm{a}$ \\
\hline & W1F1(CK) & $21.55 \pm 0.34 \mathrm{a}$ & $523.55 \pm 63.61 \mathrm{bcd}$ & $15.62 \pm 0.37 \mathrm{a}$ \\
\hline \multirow{13}{*}{2019} & DWOF0 & $24 \pm 0.58 \mathrm{abc}$ & $193.34 \pm 38.41 \mathrm{~d}$ & $18.36 \pm 0.62 \mathrm{abc}$ \\
\hline & DW0F0.5 & $24.3 \pm 0.69 \mathrm{ab}$ & $220.17 \pm 16.54 \mathrm{~cd}$ & $18.6 \pm 0.74 \mathrm{ab}$ \\
\hline & DW0F1 & $24.32 \pm 0.37 \mathrm{ab}$ & $218.57 \pm 17.69 \mathrm{~cd}$ & $18.62 \pm 0.4 \mathrm{ab}$ \\
\hline & DW0.5F0 & $24.45 \pm 1.21 \mathrm{ab}$ & $226.07 \pm 15.76 \mathrm{~cd}$ & $18.77 \pm 1.31 \mathrm{ab}$ \\
\hline & DW0.5F0.5 & $24.68 \pm 0.23 \mathrm{a}$ & $307.05 \pm 10.09 \mathrm{ab}$ & $19.02 \pm 0.25 \mathrm{a}$ \\
\hline & DW0.5F1 & $23.95 \pm 0.48 \mathrm{abc}$ & $292.73 \pm 53.9 \mathrm{abc}$ & $18.22 \pm 0.52 \mathrm{abc}$ \\
\hline & DW1F0 & $24.08 \pm 0.23 \mathrm{abc}$ & $187.34 \pm 4 \mathrm{~d}$ & $18.37 \pm 0.25 \mathrm{abc}$ \\
\hline & DW1F0.5 & $23.73 \pm 0.45 \mathrm{abc}$ & $306.34 \pm 29.75 \mathrm{ab}$ & $17.99 \pm 0.49 a b c$ \\
\hline & DW1F1 & $23.42 \pm 0.45 b c$ & $315.24 \pm 58.28 \mathrm{ab}$ & $17.65 \pm 0.48 \mathrm{bc}$ \\
\hline & DW1.5F0.5 & $23.57 \pm 0.93 b c$ & $295.86 \pm 49.76 \mathrm{abc}$ & $17.81 \pm 1.01 \mathrm{bc}$ \\
\hline & DW1.5F1 & $24.03 \pm 0.94 \mathrm{abc}$ & $349.82 \pm 69.7 \mathrm{a}$ & $18.3 \pm 1.01 \mathrm{abc}$ \\
\hline & DW1.5F1.5 & $23.55 \pm 1.38 \mathrm{bc}$ & $350.86 \pm 81.89 \mathrm{a}$ & $17.79 \pm 1.5 \mathrm{bc}$ \\
\hline & W1F1(CK) & $22.82 \pm 0.73 c$ & $251.54 \pm 21.27 \mathrm{bcd}$ & $17 \pm 0.79 c$ \\
\hline
\end{tabular}


Table 4. Cont.

\begin{tabular}{ccccc}
\hline Year & Treatment & Field Hammer & Sugarcane Stem Yield & Sucrose Content \\
\hline & DW0F0 & $23.15 \pm 0.22 \mathrm{a}$ & $387.42 \pm 60.23 \mathrm{~cd}$ & $17.36 \pm 0.24 \mathrm{a}$ \\
DW0F0.5 & $23.02 \pm 0.37 \mathrm{a}$ & $340.11 \pm 52.54 \mathrm{de}$ & $17.21 \pm 0.4 \mathrm{a}$ \\
DW0F1 & $22.97 \pm 0.38 \mathrm{a}$ & $374.44 \pm 11.68 \mathrm{de}$ & $17.16 \pm 0.41 \mathrm{a}$ \\
DW0.5F0 & $22.7 \pm 0.59 \mathrm{ab}$ & $400.62 \pm 99.43 \mathrm{bcd}$ & $16.87 \pm 0.64 \mathrm{ab}$ \\
DW0.5F0.5 & $21.85 \pm 0.96 \mathrm{~b}$ & $412.12 \pm 81.27 \mathrm{bcd}$ & $15.95 \pm 1.04 \mathrm{~b}$ \\
DW0.5F1 & $23.1 \pm 0.35 \mathrm{a}$ & $403.37 \pm 55.21 \mathrm{bcd}$ & $17.3 \pm 0.38 \mathrm{a}$ \\
DW1F0 & $23.1 \pm 0.93 \mathrm{a}$ & $281.13 \pm 59.1 \mathrm{e}$ & $17.3 \pm 1.01 \mathrm{a}$ \\
DW1F0.5 & $22.98 \pm 0.28 \mathrm{a}$ & $490.77 \pm 108.89 \mathrm{abc}$ & $17.18 \pm 0.31 \mathrm{a}$ \\
DW1F1 & $22.58 \pm 0.23 \mathrm{ab}$ & $480.72 \pm 58.1 \mathrm{abc}$ & $16.74 \pm 0.25 \mathrm{ab}$ \\
& $23.22 \pm 0.52 \mathrm{a}$ & $442.92 \pm 13.77 \mathrm{abcd}$ & $17.43 \pm 0.56 \mathrm{a}$ \\
& DW1.5F0.5 & $22.68 \pm 0.9 \mathrm{ab}$ & $501.76 \pm 38.74 \mathrm{a}$ & $16.85 \pm 0.97 \mathrm{ab}$ \\
DW1.5F1.5 & $23.22 \pm 0.08 \mathrm{a}$ & $495.48 \pm 72.91 \mathrm{ab}$ & $17.43 \pm 0.08 \mathrm{a}$ \\
W1F1(CK) & $22.97 \pm 0.33 \mathrm{a}$ & $377 \pm 40.9 \mathrm{de}$ & $17.16 \pm 0.35 \mathrm{a}$ \\
\hline
\end{tabular}

Note: Descriptions of the treatment names are given in Table 1. Data are given as the means \pm standard deviation Different letters- $a, b, c, d$ and e-in the table indicate significant differences between treatments.

One-way analysis of variance showed that there was a significant difference in sugarcane yield in $2018(\mathrm{~F}=6.877, p=0.0005)$ : sugarcane yield was the highest under the treatment of DW1F1. There was a significant difference among the treatments in 2019 $(\mathrm{F}=9.769, p<0.0001)$, where the DW1F0 treatment were the lowest, and DW1.5F1.5 was the highest. There was a significant difference in the sugarcane field hammer among the treatments in $2020(\mathrm{~F}=5.460, p=0.002)$, with DW1.5F1 being the highest and DW1F0 the lowest (Table 3). The results of the two-way ANOVA analysis showed that water had significant effect on field yield in 2018-2019, water-fertilizer interaction had significant effect on field yield in 2020 (Table 4). Considering the 3 years comprehensively, the field yield was the highest under the treatment of DW1.5F1.

One-way analysis of variance showed that there was no significant difference in sugarcane content in $2018(\mathrm{~F}=0.193, p=0.940)$ and in $2020(\mathrm{~F}=0.930, p=0.460)$, though DW1.5F0.5 was the highest in 2020 (Table 3). There was a significant difference among the treatments in $2019(\mathrm{~F}=3.802, p=0.013)$. The results of the two-way ANOVA analysis showed that water, fertilizer and water-fertilizer interaction had no significant effect on field hammer (Table 5, Figure 5).

Table 5. Results of two-way ANOVA test (F) on field hammer, sugarcane stem yield and sugar content in 2018-2020.

\begin{tabular}{cccccccccc}
\hline \multirow{2}{*}{ Treatment } & \multicolumn{3}{c}{ Field Hammer } & \multicolumn{3}{c}{ Sugar Content } & \multicolumn{3}{c}{ Sugarcane Stem Yield } \\
\cline { 2 - 9 } & $\mathbf{2 0 1 8}$ & $\mathbf{2 0 1 9}$ & $\mathbf{2 0 2 0}$ & $\mathbf{2 0 1 8}$ & $\mathbf{2 0 1 9}$ & $\mathbf{2 0 2 0}$ & $\mathbf{2 0 1 8}$ & $\mathbf{2 0 1 9}$ & $\mathbf{2 0 2 0}$ \\
\hline Water & 0.150 & $3.81 *$ & 0.93 & 0.19 & $3.82 *$ & 0.93 & $6.88^{* * *}$ & $9.77^{* *}$ & $5.46^{* *}$ \\
Fertilizer & 0.365 & 0.83 & 0.58 & 0.33 & 0.83 & 0.58 & 1.88 & $5.47^{*}$ & 2.21 \\
Water $\times$ Fertilizer & 0.576 & 0.33 & 1.81 & 0.90 & 0.55 & 1.81 & 1.22 & 1.32 & $3.62 *$ \\
\hline
\end{tabular}

Values are significant at 0.05 level; ${ }^{*}{ }^{* *}$ and ${ }^{* * *}$ refer to significant difference at $0.05,0.01$ and 0.001 probability levels, respectively. 

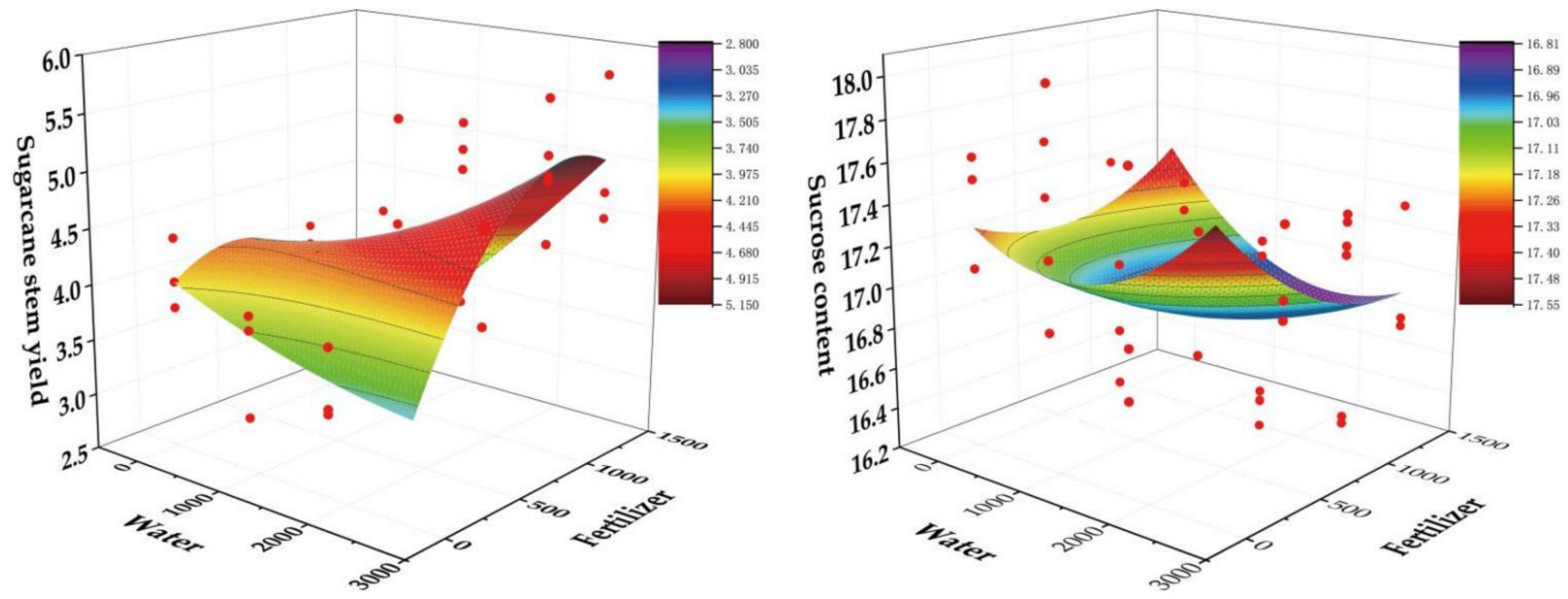

Figure 5. Regression equation of sugarcane stem yield and sucrose content with irrigation and fertilization.

\section{Discussion}

\subsection{Effects of Different Irrigation-Fertilization Combinations on Sugarcane Agronomic Traits}

Agronomic measures are known to be greatly influenced by irrigation and fertilization [29-32]. This work attempted to gain insights into the water-fertilizer coupling interaction on sugarcane growth, and thus to optimize water allocation and fertilizer application for supporting decision making in the growth of sugarcane. Previous study has shown that for the stem biomass, sugarcane needs sufficient water and moderate fertilizer at the seedling stage and jointing stage, while at the maturity stage the water needs to be reduced appropriately [33]. Among different treatments, the mean stem diameter in 3 years of DW1.5F0.5 was the highest, with an average of $27.49 \mathrm{~cm}$, followed by DW1F1, which were higher than CK (W1F1) treatment; for plant height, DW1F0.5 was highest with an average of $267.72 \mathrm{~cm}$, significantly higher than W1F1 treatment $(239.12 \mathrm{~cm})$. Effective stem numbers and stem weight were higher under DW1F1, DW1.5F1 and DW1.5F1.5 treatments, and were significantly different from the W1F1 (Figure 3). According to the nutrient and water needs of sugarcane growth stages, water-fertilizer integrated drip irrigation should be carried out to ensure effective stems, thereby maximizing sugarcane production [12].

Sugarcane absorbs a lot of nutrients throughout the growth period, and each period has a greater demand for fertilizers. The results showed that water-fertilizer integrated irrigation interaction had a significant promotion effect on sugarcane yield traits such as plant height, effective stem number and single stem weight (Table 2). The agronomic traits of sugarcane under water-fertilizer integrated drip irrigation were superior to the traditional fertilization mode (W1F1). It indicated that applying a certain amount of water and fertilizer under drip irrigation was beneficial to the growth of sugarcane [25]. The application of heavy base fertilizers with long-acting organic fertilizers and synergistic biological fertilizers was the key factor for high sugarcane yield and sugar $[34,35]$.

\subsection{Effects of Different Irrigation-Fertilization Combinations on Sugar Content and Sugarcane Yield}

Water and fertilizer have always been considered to be two major factors on agricultural production, and also as two important technical measures that can be controlled. In China, the interaction and coupling mode of water and fertilizer on dry land has always been the key topic in the multi-subject and multi-profession research. On sugarcane production, there are some reports about controlling by fertilizer or water [34,35] to improve the growth, yield, quality and economic benefits of sugarcane, and some reports about the coupling effects of different levels of fertilization and irrigation [36,37]. 
To meet the nutritional requirements of sugarcane growth, make sugarcane grow normally and to obtain sugarcane's high yield and high-quality raw sugarcane is our object. In this experiment, by setting low, medium and high irrigation and fertilization level treatments, it is confirmed that water will affect the yield of sugarcane (Table 5). The combination of heavy fertilizer with water is the key factor for sugarcane's high yield (Table 4). However, for sugar content, the most beneficial combined treatment was W0.5F0, which means the sugar content was higher under the treatment of low water and fertilization coupling, but the yield of sugarcane was not high under this treatment. Inman-Bamber et al clearly demonstrated that reducing irrigation during the maturation phase increases the sucrose content in sugarcane, and it is now common practice in irrigated sugarcane production to suspend irrigation before harvesting [11]. Combined with the sugar content and the final yield of sugarcane in this study, the most suitable coupling treatment of water and fertilizer was DW1F1, that is, too much water and fertilizer is not necessarily conducive to the economic benefit of sugarcane.

Compared with the traditional manpower irrigation and fertilization, drip irrigation was beneficial for stem growth and appropriate for field planting. From the angle of sustainable development, the coupling of water and fertilizer is the only way to strive for high yield and high efficiency sugar on sugarcane [35-41]. Combining high fertilizer doses with irrigation by methods that are more tolerant to water deprivation can save on irrigation costs and improve sugar yield. The best treatment for sugarcane yield was to combine medium water with high fertilization.

\section{Conclusions}

Water and fertilizer during the co-growth period are the major factors limiting the productivity of the sugarcane in Guangxi, China. Over-irrigation and fertilization is primarily a waste of resources and also causes an unfavorable impact on the natural environment. Therefore, research is needed to find the most appropriate level of soil moisture and nutrient content to obtain the highest production efficiency in sugarcane cultivation. Therefore, it is crucial to optimize the management of water and fertilizer in the region. Based on the relationships observed between water and fertilization inputs, dray irrigation supplying $1778 \mathrm{~m}^{3} \mathrm{ha}^{-1}$ and a fertilizer dose of $933 \mathrm{~kg} \mathrm{ha}^{-1}$ maximized sugarcane yield. Going forward, the coupling of water and fertilizer should be carried out in relation to different stages of sugarcane. In addition, the combined use of advanced drip irrigation, sprinkler irrigation and a pipeline system with water supply irrigation facilities will help to improve the use efficiency of water and nutrients, improving sugarcane yield and sugar content in sugarcane production in South of China, which are very important to promote sugarcane industry development, improve utilization efficiency of water and fertilizer resources, increase sugarcane farmers' income, reduce sugarcane fertilizer, water and soil loss and to solve the sharp contradiction of high-yield, high-efficiency production and environmental protection.

Author Contributions: Conceptualization, W.W. and Y.B.; methodology, Y.B.; software, Z.M.; validation, J.M.A. and W.F.; formal analysis, W.F.; investigation, Z.M.; resources, W.W.; data curation, Z.M.; writing-original draft preparation, Z.M.; writing-review and editing, J.M.A.; visualization, Z.M.; supervision, Y.B.; project administration, Y.B.; funding acquisition, W.W. All authors have read and agreed to the published version of the manuscript.

Funding: This research was funded by Guangxi Key R\&D Program, grant number Guike AB19245040. Institutional Review Board Statement: Not applicable.

Informed Consent Statement: Not applicable.

Data Availability Statement: This study did not report any data.

Acknowledgments: The authors thank Guangxi Key R\&D Program (Guike AB19245040) assisted with data presentation. 
Conflicts of Interest: The authors declare no conflict of interest.

\section{References}

1. Arro, J.; Park, J.W.; Wai, C.M.; VanBuren, R.; Pan, Y.B.; Nagai, C.; Da Silva, J.; Ming, R. Balancing selection contributed to domestication of autopolyploid sugarcane (Saccharum officinarum L.). Euphytica 2016, 209, 477-493. [CrossRef]

2. Abrar, T.; Albejo, A. Sugarcane (Saccharum officinarum L.) Tissue Culture in Ethiopia: Opportunities for Ethiopia's Sugar Industries. Int. J. Sci. Technol. Res. 2017, 6, 398-406.

3. Singh, H.; Singh, R.; Kumar, V.; Meena, R. Nitrogen fertigation schedule and irrigation effects on productivity and economics of spring sugarcane. Indian J. Agric. Res. 2019, 53, 405-410. [CrossRef]

4. Singh, K.; Rinwa, R.; Kumar, P.; Rani, S. Growth, Nutrient Use Efficiency and Quality of Spring Sugarcane (Saccharum officinarum L.) under Different Planting Method and Nitrogen Level. Agric. Sci. Digest 2019, 39, 324-327. [CrossRef]

5. Meng, S.; Wang, X.; Huang, G.; Lu, Y.; Huang, P.; Lu, G. Different Fertilization Modes of Sugarcane in Latosolic Red Soil of Guangxi. Asian Agric. Res. 2019, 11,77-83.

6. Lakshmanan, P.; Robinson, N. Stress Physiology: Abiotic Stresses. In Sugarcane: Physiology, Biochemistry, and Functional Biology; Wiley Blackwell: Ames, IA, USA, 2013; pp. 411-434. [CrossRef]

7. Marin, F.R.; Inman-Bamber, G.; Silva, T.G.F.; Vianna, M.S.; Nassif, D.S.P.; Carvalho, K.S. Sugarcane evapotranspiration and irrigation requirements in tropical climates. Theor. Appl. Climatol. 2020, 140, 1349-1357. [CrossRef]

8. Machado, R.S.; Ribeiro, R.V.; Marchiori, P.E.R.; Machado, D.F.S.P.; Machado, E.C.; Landell, M.G.d.A. Respostas biométricas e fisiológicas ao deficit hídrico em cana-de-açúcar em diferentes fases fenológicas. Pesqui. Agropecu. Bras. 2009, 44, 1575-1582. [CrossRef]

9. Roberts, J.; Nayamuth, R.A.; Batchelor, C.H.; Soopramanien, G.C. Plant-water relations of sugarcane (Saccharum officinarum L.) under a range of irrigated treatments. Agric. Water Manag. 1990, 17, 95-115. [CrossRef]

10. Robertson, F.A.; Thorburn, P.J. Management of sugarcane harvest residues: Consequences for soil carbon and nitrogen. Soil Res. 2007, 45, 13-23. [CrossRef]

11. Inman-Bamber, N.G.; Smith, D.M. Water relations in sugarcane and response to water deficits. Field Crops Res. 2005, 92, 185-202. [CrossRef]

12. Olivier, F.C.; Singels, A. Increasing water use efficiency of irrigated sugarcane production in South Africa through better agronomic practices. Field Crops Res. 2015, 176, 87-98. [CrossRef]

13. Chen, Y.L.; Feng, L.P.; Ding, M.H.; Mo, W.H.; Kuang, Z.M. Effects of Meteorological Factors on Stalk Elongation in New-planting and Ra-tooning Sugarcane. Acta Agron. Sin. 2016, 42, 583-590. [CrossRef]

14. Wiedenfeld, R.P. Water stress during different sugarcane growth periods on yield and response to N fertilization. Agric. Water Manag. 2000, 43, 173-182. [CrossRef]

15. Franco, H.; Castro, S.; Sanches, G.; Kölln, O.; Bordonal, R.; Borges, C.; Melo Montes Nogueira Borges, B. Alternatives to Increase the Sustainability of Sugarcane Production in Brazil under High Intensive Mechanization. In Sustainable Sugarcane Production, 1st ed.; Singh, P., Tiwari, A.K., Eds.; CRC Press: Waretown, NJ, USA, 2007; Volume 3, pp. 154-196.

16. Franco, H.C.J.; Otto, R.; Faroni, C.E.; Vitti, A.C.; Almeida de Oliveira, E.C.; Trivelin, P.C.O. Nitrogen in sugarcane derived from fertilizer under Brazilian field conditions. Field Crops Res. 2011, 121, 29-41. [CrossRef]

17. Thorburn, P.J.; Biggs, J.S.; Jeda, P.; Meier, E.A.; Kirsten, V.; Skocaj, D.M. Prioritizing Crop Management to Increase Nitrogen Use Efficiency in Australian Sugarcane Crops. Front. Plant Sci. 2017, 8, 1504. [CrossRef]

18. Cortez, L.A.B. Sugarcane bioethanol. In Sugarcane Bioethanol: $R$ \& D for Productivity and Sustainability; Blucher: Sao Paulo, Brazil, 2010; pp. 405-422.

19. Oweis, T.Y.; Farahani, H.J.; Hachum, A.Y. Evapotranspiration and water use of full and deficit irrigated cotton in the Mediterranean environment in northern Syria. Agric. Water Manag. 2011, 98, 1239-1248. [CrossRef]

20. McConnell, J.S.; Wilkerson, M.H.; Mitchell, G.A. Influences of nitrogen treatments and irrigation methods on soil chemical properties. Commun. Soil Ence Plant Anal. 1988, 19, 1925-1943. [CrossRef]

21. Lv, H.; Zhao, Y.; Wang, Y.; Li, W.; Wang, J.; Butterbach-Bahl, K.; Lin, S. Conventional flooding irrigation and over fertilization drives soil $\mathrm{pH}$ decrease not only in the top-but also in subsoil layers in solar greenhouse vegetable production systems. Geoderma 2020, 363, 114156. [CrossRef]

22. Gleeson, T.; Wang-Erlandsson, L.; Zipper, S.C.; Porkka, M.; Jaramillo, F.; Gerten, D.; Fetzer, I.; Cornell, S.E.; Piemontese, L.; Gordon, L.J.; et al. The Water Planetary Boundary: Interrogation and Revision. One Earth 2020, 2, 223-234. [CrossRef]

23. Al-Ghobari, H.M. The impact of excessive irrigation water and fertilizers on the environment and groundwater quality in arid regions. In Environmental Impact; Brebbia, C.A., Ed.; WIT Press: Southampton, UK, 2012; Volume 162, pp. 313-323.

24. Sahay, S.; Iqbal, S.; Inam, A.; Gupta, M.; Inam, A. Waste water irrigation in the regulation of soil properties, growth determinants, and heavy metal accumulation in different Brassica species. Environ. Monit. Assess. 2019, 191, 107. [CrossRef]

25. Tan, Z.K.; Ou, Z.R.; He, Y. Analysis of the Major Meteorological Calamity and the Research on Optimum Layout of Cane Sugar Production in Guangxi. Sugarcane Canesugar 2006, 1, 17-21.

26. Lu, Y.F.; Liao, X.P.; Chen, X.; Li, Y.X.; Li, Y.Z. The spatial-temporal characteristics of sugarcane growing season drought during 40 years in Youjiang River Valley area of Guangxi. J. Meteorol. Res. Appl. 2015, 36, 62-65. 
27. Denmead, O.T.; Macdonald, B.C.T.; Bryant, G.; Naylor, T.; Wilson, S.; Griffith, D.W.T.; Wang, W.J.; Salter, B.; White, I.; Moody, P.W. Emissions of methane and nitrous oxide from Australian sugarcane soils. Agric. For. Meteorol. 2010, 150, 748-756. [CrossRef]

28. CSY. Guangxi Statistical Yearbook. China Statistical Publishing House, Beijing 2010-2019. 2019. Available online: http: / / tjj.gxzf.gov.cn/ / tjsj/tjnj/material/tjnj20200415/2020/zk/indexch.htm (accessed on 1 December 2021).

29. Comas, L.H.; Trout, T.J.; Dejonge, K.C.; Zhang, H.H.; Gleason, S.M. Water productivity under strategic growth stage-based deficit irrigation in maize. Agric. Water Manag. 2018, 212, 433-440. [CrossRef]

30. Finch, T. Restoring farmlands for food and nature. One Earth 2020, 3, 665-668. [CrossRef]

31. Herzog, T. World Greenhouse Gas Emissions in 2005. WRI Working Paper. World Resources Institute, 2009. 2005. Available online: http:/ / www.wri.org/publication/navigating-the-numbers (accessed on 1 December 2021).

32. Wang, E.; Attard, S.; Linton, A.; McGlinchey, M.; Xiang, W.; Philippa, B.; Everingham, Y. Development of a closed-loop irrigation system for sugarcane farms using the Internet of Things. Comput. Electron. Agric. 2020, 172, 105376. [CrossRef]

33. Ma, Z.X.; Wu, W.X.; Alatalo, J.M.; Fu, W.X.; Bai, Y. Optimal Water-Fertilizer Combinations for Efficient Nitrogen Fixation by Sugarcane at Different Stages of Growth. Water 2021, 13, 2895. [CrossRef]

34. Wu, L.; Zhang, F.C.; Fan, J.L.; Zhou, H.M.; Liang, F.; Gao, Z.J. Effects of Water and Fertilizer Coupling on Cotton Yield, Net Benefits and Water Use Efficiency. Trans. Chin. Soc. Agric. Mach. 2015, 46, 164-172.

35. Liu, Z.; Li, J.H.; Yao, Y.X.; Wang, P.; Xu, T.W. Experiment on water-consumption rule of sugarcane under condition of regulated deficit irrigation. J. Water Resour. Water Eng. 2011, 22, 173-175.

36. Tan, H.W.; Liu, Y.X.; Zhou, L.Q.; Xie, R.L.; Yang, S.D.; Huang, J.S.; Huang, M.F. Reduction Fertilization Techonogies under Drip for Sugarcane Irrigation. Chin. J. Trop. Crops 2013, 34, 024-028.

37. Zheng, S.P.; Liu, Z.X. Sustainable Agriculture in Semiarid Region and Interaction between Water and Fertilizer. Res. Agric. Mod. 2000, 21, 291-294.

38. Wen, H.D.; Liu, Y.Z.; Li, X.L.; Li, S.W.; Wang, D.W. Water and fertilizer coupling and dryland agricultural sustainable development. Soil Environ. Sci. 2002, 11, 315-318.

39. Shen, H.; Chen, Y.; Hu, Y.; Ran, L.; Lam, S.K.; Pavur, G.K.; Zhou, F.; Pleim, J.E.; Russell, A.G. Intense Warming Will Significantly Increase Cropland Ammonia Volatilization Threatening Food Security and Ecosystem Health. One Earth 2020, 3, 126-134. [CrossRef]

40. Silva, W.; Neves, T.; Silva, C.; Carvalho, M.; Abraho, R. Sustainable enhancement of sugarcane fertilization for energy purposes in hot climates. Renew. Energy 2020, 159, 547-552. [CrossRef]

41. Silva, T.G.F.d.; Moura, M.S.B.d.; Zolnier, S.; Souza, L.S.B.d. Biomassa seca acumulada, partições e rendimento industrial da cana-de-açúcar irrigada no Semiárido brasileiro. Rev. Ceres 2014, 61, 686-696. [CrossRef] 